\title{
A Steady FastICA Algorithm Based on Modified-M Estimate Function
}

\author{
Shexiang $\mathrm{Ma}^{+}$and Ping Xie \\ School of Computer and Communication Engineering, Tianjin University of Technology, Tianjin, China
}

\begin{abstract}
In order to improve the detection rate of ship information, this paper adopts fast independent component analysis (FastICA) algorithm to separate satellite-based automatic identification system (AIS) mixed signals. FastICA algorithm has the advantages of fast convergence rate and simple form. However, it is sensitive to the initialization of the separation matrix and the robust performance is poor. Aimed at the problem, this paper proposes an improved FastICA algorithm. This new algorithm is based on constant model of AIS signal and chooses Modified-M estimation function as nonlinear function so as to improve the robustness of algorithm. The improved algorithm also modifies Newton iterative algorithm. The experimental simulation results indicate that the proposed method reduces the iteration times, the accuracy has been improved.
\end{abstract}

Keywords: satellite-based automatic identification system, fast independent component analysis, robustness, modified-M estimate function.

\section{Introduction}

As a new kind of ship navigation system, AIS can be used for data transmission between ships and shore units [1]. Satellite-based AIS receives the signals through the satellite and feedbacks the information to the test center of the shore [2]. Then we can realize a global monitoring of the ship. Usually, satellite-based AIS communication range is more than 1500 nautical mile (radius), within the same satellite view contains a plurality of AIS subnets. And this will cause the signal collision phenomenon which can reduce ship detection rate, thus how to separate signal accurately is an important issue.

Since the source signal (i.e. a signal transmitted by AIS subnet) is statistically independent, this paper adopts the method of ICA [3] to separate the AIS mixed signal. ICA algorithm has been widely used in various subjects, such as speech signal processing, medical signal processing, image enhancement, pattern recognition. It includes information maximization (Informax) algorithm [4] and extended Informax [5], the natural gradient algorithm [6] and FastICA algorithm [7] and so on. Among them Informax algorithm is only used in the situation with super-Gaussian distribution. Then the extended Infomax algorithm can separate super-Gaussian and sub-Gaussian mixed signal, but its convergence rate is slow. The natural gradient algorithm is only applicable to the case that the number of observed signals is equal to the number of source signals. FastICA algorithm, also known as the fixed point algorithm, can estimate independent components only through a nonlinear function. However its robustness is not good and it is sensitive to the initial value of the weight vector. To solve this problem, Douglas S C and Chao J C put forward an improved FastICA algorithm, using Huber $\mathrm{M}$ estimation function as a nonlinear function. And Jun-an YANG et al. proposed a FastICA algorithm, which combines the Newton iterative method with gradient descent method. In this paper, an improved algorithm is proposed based on the constant model of AIS signal.

\section{The Original FastICA Algorithm}

\footnotetext{
Corresponding author. Tel.: + 18920587998

E-mail address: masx_tjut@126.com.
} 


\subsection{Signal Separation Model}

ICA technique separates mixed signals blindly without any information of mixing system. Depending on the mixing process, the blind separation can be divided into linear instantaneous mixing, linear convolution mixing and nonlinear mixing, etc. For satellite-based AIS, received signal is linear instantaneous mixing way, the separation model as illustrated in Fig. 1.

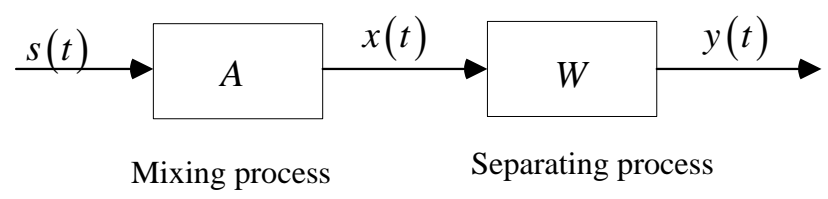

Fig. 1: The separation model.

In the Fig.1, $s(t)=\left[\mathrm{s}_{1}(t), \mathrm{s}_{2}(t), \cdots, \mathrm{s}_{N}(t)\right]^{T}$ shows $N$ dimensional statistically independent signal sources $x(t)=\left[x_{1}(t), x_{2}(t), \cdots, x_{M}(t)\right]^{T}$ denotes $M$ dimensional observed signals, $y(t)=\left[y_{1}(t), y_{2}(t), \cdots, y_{N}(t)\right]^{T}$ means separation signals. So mathematical model of the ICA can be expressed by matrix:

$$
X=A S
$$

In the formula, $A$ is $M \times N$ dimensional mixing matrix and it represents unknown transmission channel. Number of independent components here is assumed to be equal to the number of observed mixtures, which is $M=N$. The task of blind source separation is to identify the signal sources by estimating the de-mixing matrix $W$. Therefore, the separation signal is expressed as:

$$
Y=W X=W A S
$$

\subsection{Signal Preprocessing}

When conducting the signal separation in FastICA algorithm, we often do preprocessing for the observed signal. Processing includes signal centering and pre-whitening. The centering process is to use time average to replace statistic average, using the arithmetic mean value to replace mathematical expectation. For the convenience of discussion, it is considered that $X$ is random variable with mean value of zero. In the separation question, conduct whitening for $X$ through a linear transformation $B$, so $Z=B X$ can satisfy:

$$
R_{z}=E\left[z z^{T}\right]=I
$$

In the formula, $I$ is a unit matrix, $B$ means the whitening matrix and $Z$ expresses the whiten signal. So:

$$
R_{z}=E\left[(B X)(B X)^{T}\right]=B R_{X} B^{T}=I
$$

Because $R_{X}$ is generally symmetrical and non-negative definite, it can be calculated by the principal component analysis as follows:

$$
B=D^{-\frac{1}{2}} U^{T}
$$

where, $U$ is the characteristic vector matrix of $R_{X}, D$ is the diagonal matrix of corresponding characteristic value. Above all, signal preprocessing makes the observed signal into unrelated components, and this can reduce the workload of the algorithm.

\subsection{Component Extraction Processing}

FastICA algorithm includes data processing and component extraction processing. Among them, the independent component extraction is realized by constructing and optimizing the objective function. In the paper, the objective function is based on negative entropy and its form as follows:

$$
\begin{gathered}
J_{G}(w)=E\left\{G\left(w^{T} Z\right)\right\}-\beta\left(E\left\{\left(w^{T} Z\right)^{2}\right\}-1\right) \\
\beta=E\left\{w_{0}^{H} Z \cdot g\left(w_{0}^{H} Z\right)\right\}
\end{gathered}
$$


Here $G(\cdot)$ is nonlinear function, and it is desirable as $G(y)=\ln \cdot \cosh (y) \operatorname{combined}$ with the AIS signal non-Gaussian characteristics, $g(\cdot)$ is the differential coefficient of $G(\cdot), w_{0}$ is the initial value of $w$.Use Newton iterative method with a locally quadratic convergence rate to optimize the objective function. The Newton iterative formula is given below:

$$
w_{k+1}=w_{i}-\frac{F\left(w_{k}\right)}{F^{\prime}\left(w_{k}\right)}
$$

where $k$ is the number of iterations, and $F(w)=J_{G}(w)$, there are:

$$
\begin{gathered}
F(w)=E\left\{Z \cdot g\left(w^{T} Z\right)\right\}-\beta w \\
F^{\prime}(w)=E\left\{g^{\prime}\left(w^{T} Z\right)\right\}-\beta I
\end{gathered}
$$

Put the equation (8) (9) into equation (7), we can get the iterative formula as follows:

$$
w_{k+1}=E\left\{Z g\left(w_{k}^{T} Z\right)\right\}-E\left\{g^{\prime}\left(w_{k}^{T} Z\right)\right\} w_{k}
$$

When the convergence condition $\left|w_{k+1}-w_{k}\right| \leq \delta$ ( $\delta$ is the convergence threshold) is fulfilled, make $w=w_{k+1}$ and extract the signal $y_{i}=w^{T} Z$.

\section{The Improved FastICA Algorithm}

The performance of FastICA algorithm is determined by the initial condition $w_{0}$ and the nonlinear function $G(\cdot)$.At the same time, the convergence speed is affected by the Newton iteration algorithm. Therefore, this paper improves the FastICA algorithm based on these three areas. Firstly, AIS signal is a GMSK modulation signal with constant module feature, hence constant modulus character can be used to blind source separation to improve the FastICA algorithm. So we calculate the initial weight vector through stochastic gradient constant modulus algorithm. Define the cost function as shown below:

$$
J(\hat{w}(k))=E\left\{\left.|| \hat{w}^{H}(k) x(k)\right|^{p}-\left.|\alpha|^{p}\right|^{q}\right\}
$$

where, $\alpha$ is amplitude of a desired signal, $p$ and $q$ are positive integer, which can affect the convergence property and complexity. Let be $\alpha=1 p=1$ and $q=2$, iterative formula can be deduced:

$$
\hat{w}(\mathrm{k}+1)=\hat{w}(k)-\mu x(k) e^{*}(k)
$$

where, $e(k)=2\left[\hat{w}^{H}(k) x(k)-\frac{\hat{w}^{H}(k) x(k)}{\left|\hat{w}^{H}(k) x(k)\right|}\right], \mu=\frac{1}{2 \lambda_{1}^{2}}$ is the step factor, $\lambda_{1}$ is the biggest non-zero singular value of receiving data matrix.

The second step of the improved algorithm, we select Modified-M estimation function [8] as a nonlinear function of the objective function, which is expressed as:

$$
G(u)=\left\{\begin{array}{cc}
\frac{1}{2} u^{2}, & |u| \leq a \\
a|u|-\frac{1}{2} a^{2}, & a<|u| \leq b \\
\frac{a}{b} \frac{1-e^{c(-|u|+b)}(1+c|u|)}{c^{2}}+a b-\frac{1}{2} a^{2}+\frac{a}{c}, & |u|>b
\end{array}\right.
$$

where, $c$ is the impact factor, $a$ and $b$ are the boarders. When $a=1.345 b=3 c=0.3$, the robustness is best.

Finally, we amend the second-order Newton iterative algorithm for the third-order Newton iterative algorithm so as to improve the convergence speed. The specific form is shown as follows: 


$$
\begin{gathered}
\tilde{w}_{k+1}=w_{k}-\frac{F\left(w_{k}\right)}{F^{\prime}\left(w_{k}\right)} \\
w_{k+1}=w_{k}-\frac{2 F\left(w_{k}\right)}{F^{\prime}\left(w_{k}\right)+F^{\prime}\left(\tilde{w}_{k+1}\right)}
\end{gathered}
$$

After calculation, the iterative formula of improved algorithm can be simplified to:

$$
\begin{gathered}
\tilde{w}_{k+1}=E\left\{Z g\left(w_{k}^{T} Z\right)\right\}-E\left\{g^{\prime}\left(w_{k}^{T} Z\right)\right\} w_{k} \\
w_{k+1}=2 E\left\{Z g\left(w_{k}^{T} Z\right)\right\}-\left\{E\left\{g^{\prime}\left(w_{k}^{T} Z\right)\right\}+E\left\{g^{\prime}\left(\tilde{w}_{k}^{T} Z\right)\right\}\right\} w_{k}
\end{gathered}
$$

In summary, the entire improved FastICA algorithm is described as follows:

(1)Preprocessing for the observed signals;

(2)Calculating the initial weight vector through stochastic gradient constant modulus algorithm;

(3)Constructing the objective function combined with the initial weight vector and Modified-M estimate function;

(4)Optimizing the objective function by the third-order Newton iteration algorithm so that the signal $y_{i}$ can be separated;

(5)If $i$ is less than $N$, remove the correlation of separating vector. Then repeat step (2) until $i=N$.

\section{Experimental Results}

\subsection{Performance Evaluation}

In this paper, we use the similarity coefficient to evaluate the separation efficiency of blind source separation algorithm. Assume that $s_{i}$ is one source signal and $y_{j}$ is the estimated signal of $s_{i}$. The similarity coefficient can be described as:

$$
\rho=\frac{\left|\operatorname{cov}\left(s_{i}, \mathrm{y}_{j}\right)\right|}{\sqrt{\operatorname{cov}\left(s_{i}, s_{i}\right) \operatorname{cov}\left(\mathrm{y}_{j}, \mathrm{y}_{j}\right)}}
$$

In the formula, the value of $\operatorname{cov}(\bullet)$ is the covariance. As a result of the separation sequence uncertainty, $i$ and $j$ are not necessarily the same. And the more close the value of similarity coefficient $\rho$ is to 1 , the greater the correlation of signals is. In order to show the performance of separation method more accurately, we also choose mean square error SMSE as the evaluation criteria. Assume that $N$ is the number of the signal sources, SMSE can be obtained as:

$$
S M S E=\frac{1}{N} \sum_{i=1}^{N} E\left\{\left|s_{i}-y_{j}\right|^{2}\right\}
$$

We can get that the smaller the value of SMSE, the better separation effect of the algorithm. When SMSE is equal to zero, estimated signal is no difference in full with the source signal.

\subsection{Simulation Experiment}

This paper performs some signal simulation experiments under the environment of Matlab. Here, AIS source signal is GMSK modulation, bit-rate is $9600 \mathrm{bit} / \mathrm{s}$, carrier frequency is $40 \mathrm{kHz}$. Mix 4 source signals through a mixing matrix $A$, then adopt the improved algorithm to estimate source signal. The simulation results as follows.

Through the Figs. 2, 3, 4, 5 experimental results, it can be seen that all the four algorithms can extract signals from the mixed signal completely. Fig. 6 simulates the mean square error curve for the four algorithms. From the graph, it can be seen that the signal mean square error of the improved algorithm in this 
paper is below the other three algorithms. Therefore, the algorithm of this text is superior to the other three algorithms in this paper.

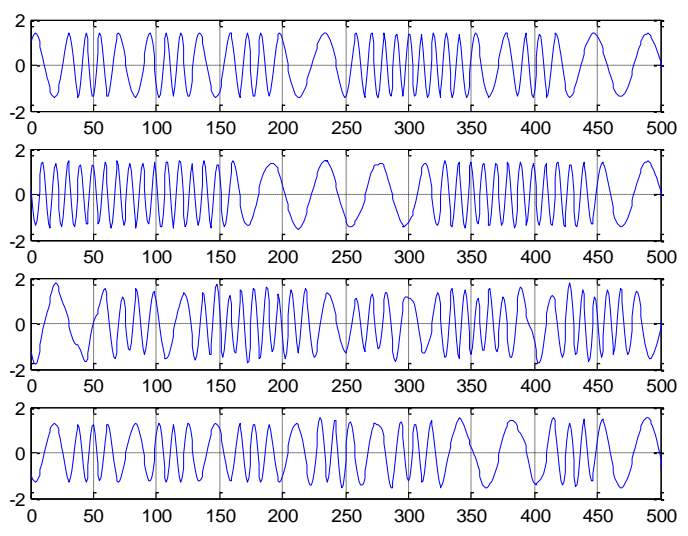

Fig. 2: Separated signals by FastICA algorithm.
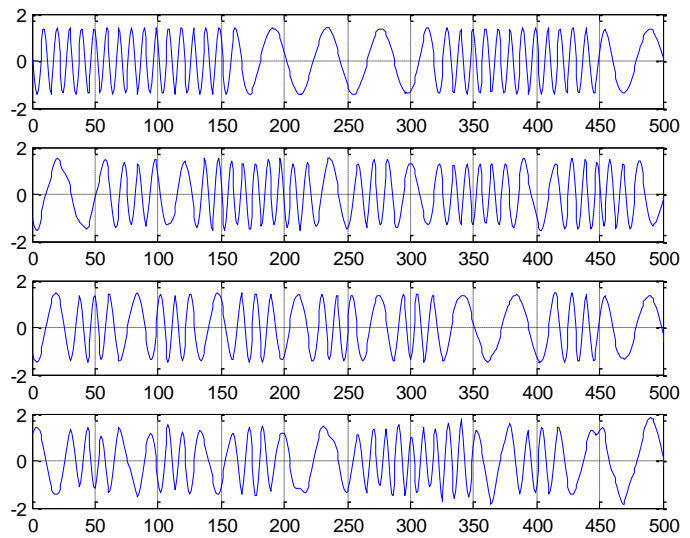

Fig. 4: Separated signals by document [10] algorithm.

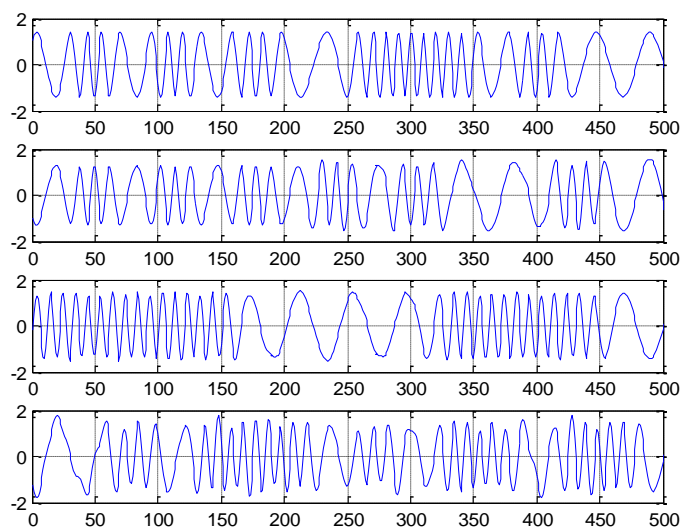

Fig. 3: Separated signals by document [9] algorithm.

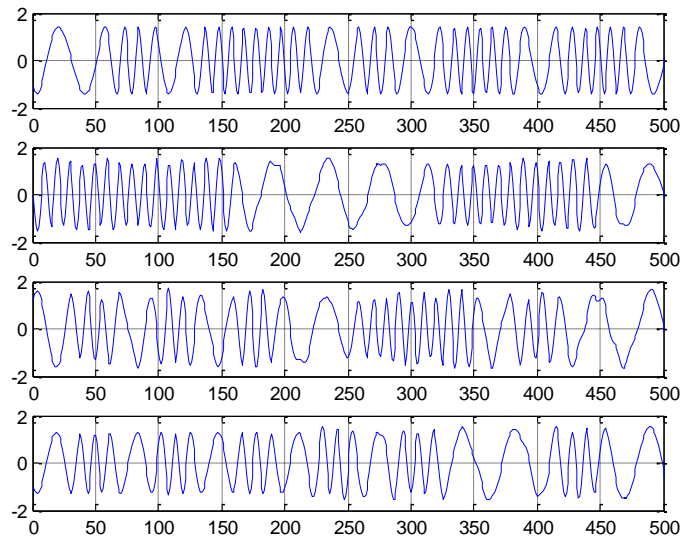

Fig. 5: Separated signals by proposed algorithm.

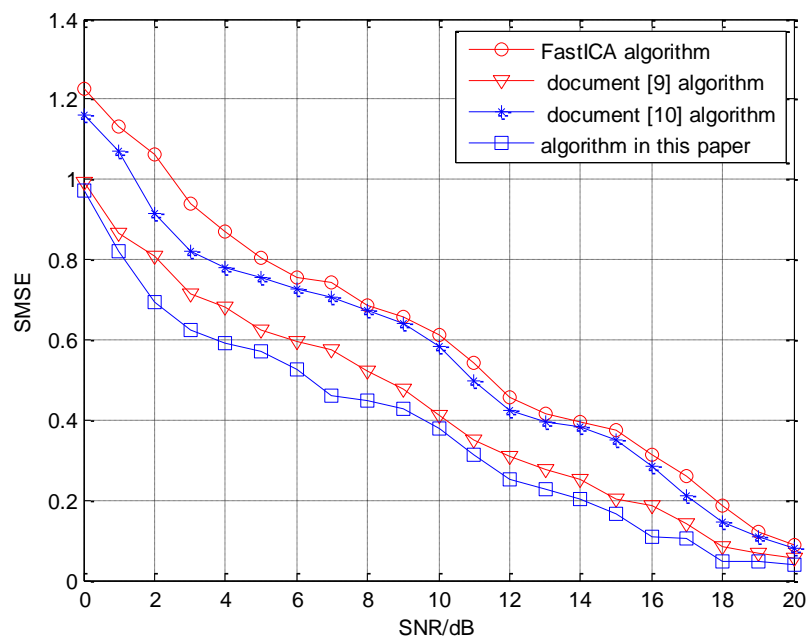

Fig. 6: The mean square error curves of four algorithms.

Table 1 shows the iteration times of four algorithms. It can be seen that when the number of source signals is 2 , the iteration times is so little difference between the four algorithms. With the increase of the number of source signals, the iteration times of the improved algorithm is much smaller than that of the other three algorithms. Hence the method in this paper can speed up the convergence rate and reduce the number of iterations. 
Table 1: The Iteration Times of four Algorithms

\begin{tabular}{|c|c|c|c|c|}
\hline $\begin{array}{c}\text { The number of } \\
\text { source signals }\end{array}$ & 2 & 6 & 10 & 15 \\
\hline FastICA algorithm & 12 & 98 & 210 & 442 \\
\hline document[9] algorithm & 10 & 66 & 140 & 251 \\
\hline document[10] algorithm & 10 & 53 & 122 & 223 \\
\hline algorithm in this paper & 10 & 39 & 89 & 167 \\
\hline
\end{tabular}

In this paper, we proposed an improved FastICA algorithm with Modified-M function for overcome the disadvantage of the original algorithm. Simulation results show that the method can separate signals effectively. In the same signal to noise ratio, the mean square error of the new algorithm is smaller than the other three algorithms'. In addition improved algorithm accelerates the convergence rate, reduces the number of iterations. Hence, the improved FastICA algorithm of the paper has a good improvement in separation performance and convergence speed.

\section{Conclusion}

In this paper, the research results are obtained by simulation data. In a follow-up study, we should validate feasibility of the algorithm through experiments with real data. And I want to thank my tutor specifically_-Professor Ma Shexiang. He provides valuable advice and suggestions to my research. His earnest research attitude and continuous efforts of life is worth learning. By the end of this paper, I would like to express our heartfelt thanks to Professor Ma.

\section{Acknowledgment}

This project was supported by the National Nature Science Foundation of China (No.61371108) and High School Science and Technology Fund Planning Project of Tianjin (No.20140706, No.20140707).

\section{References}

[1] Challamel R. A European hybrid high performance Satellite-AIS system. Advanced Satellite Multimedia Systems Conference, Baiona: IEEE Press, 2012:246-252.

[2] Cervera MA, Ginesi A, Eckstein K. Satellite-based vessel Automatic Identification System: a feasibility and performance analysis. International Journal of Satellite Communications and Network, 2011, 29(2):117-142.

[3] Choi S, Cichocki A, Park H M, Lee S Y. Blind source separation and independent component analysis: A review. Neural Inf. Process, 2005, 6(1):1-57.

[4] Bell A J, Sejnowski T J. An information-maximization approach to blind separation and blind de-convolution. Neural Computation, 1995, 7(6):1129-1159.

[5] Liu D, Liu X. Informax algorithm based on linear ICA neural network for BSS problems. Processing of the 4th World Congress on Intelligent Control and Automation, 2002:1949-1952.

[6] Lei Li, Fei Yan. A New Independent Component Analysis Based on Extended-Natural Gradient. IEEE: the International Conference on Machine Learning and Cybernetics, 2007:2416-2420.

[7] Zarzoso V, Comon P. Comparative speed analysis of FastICA. Processing of the 7th International Conference on Independent Component Analysis and Signal Separation, 2007:293-300.

[8] Wang Bo. Research and application of statistical data quality assessment methods. Master's Thesis of North China Electric Power University, 2013.

[9] Douglas S C, Chao J C. Simple, robust, and memory-efficient FastICA algorithms using the Huber M-estimator cost function. The Journal of VLSI Signal Processing, 2007, 48(1-2):143-159.

[10] Yang Jun-an, Zhuang Zhen-quan, Wu Bo. An improved FastICA algorithm based on negentropy maximization. Journal of Circuits and Systems, 2002, 7(4):37-40. 